\title{
Difficulty in management of intraductal papillary mucinous neoplasm-associated pancreatobiliary fistulas and the role of "pig-nose" appearance and intraductal ultrasonography in diagnosis
}

Authors

Institutions
Mitsuhito Koizumi ${ }^{1}$, Teru Kumagi ${ }^{1}$, Taira Kuroda ${ }^{1}$, Nobuaki Azemoto ${ }^{1}$, Hirofumi Yamanishi ${ }^{1}$, Yoshinori Ohno ${ }^{1}$, Tomoyuki Yokota ${ }^{1,2}$, Hironori Ochi ${ }^{2}$, Kazuhiro Tange $^{2}$, Yoshiou keda $^{3}$, Yoichi Hiasa ${ }^{1}$

${ }^{1}$ Gastroenterology and Metabology, Ehime University Graduate School of Medicine, Ehime, Japan

${ }^{2}$ Center for Liver-Biliary-Pancreatic Diseases, Matsuyama Red Cross Hospital, Matsuyama, Japan

${ }^{3}$ Endoscopy Center, Ehime University Hospital, Ehime, Japan submitted

24. December 2015

accepted after revision

8. February 2016

\section{Bibliography}

DOI http://dx.doi.org/

10.1055/s-0042-102956

Published online: 30.3.2016

Endoscopy International Open

2016; 04: E446-E450

(c) Georg Thieme Verlag KG

Stuttgart · New York

E-ISSN 2196-9736

\section{Corresponding author}

Teru Kumagi

Gastroenterology and

Metabology

Ehime University Graduate

School of Medicine

Shitsukawa

Toon

Ehime 791-0295

Japan

Fax: +81-89-9605310

terukuma@m.ehime-u.ac.jp

\section{License terms}

(ब) (1) $\Theta \circledast$
Pancreatobiliary fistulas associated with intraductal papillary mucinous neoplasms (IPMN) often develop obstructive jaundice and cholangitis; thus, early diagnosis is important. However, computed tomography and cholangiography, the current methods for detecting pancreatobiliary fistulas, are not always effective. We previously reported a case of IPMN-associated pancreatobiliary fistula and proposed a potential new diagnostic marker: the "pig-nose" appearance of the duodenal papilla, which results from dilated pancreatic and bile ducts and can be visualized via endoscopy. In this study, we report another three cases of IPMN-associated pancreatobiliary fistulas detected by a different technology, intraductal ul-

\section{Introduction}

$\nabla$

Since the first reported case in 1982, the intraductal papillary mucinous neoplasm (IPMN) has become a distinct clinical pathologic entity and is increasingly being recognized globally [1]. IPMN occasionally involves formation of fistulas with surrounding organs, including bile ducts $[2,3]$. Previous reports implicate two factors in fistula pathogenesis: (1) direct invasion of IPMN into adjacent organs, and (2) pancreatic perforation due to inflammatory stimulation and high pressure in pancreatic ducts [2]. Pancreatobiliary fistulas associated with IPMN must be diagnosed early because they often develop obstructive jaundice and cholangitis. Before surgery, most IPMN-associated pancreatobiliary fistulas are identified by cholangiography or computed tomography (CT) [3]. However, because these methods sometimes fail to detect pancreatobiliary fistulas [4,5], alternative technologies are needed. Intraductal ultrasonography (IDUS) is a candidate technology in this regard because it visualizes the common bile duct (CBD) wall and its surrounding structures with high resolution. We have previously introduced a unique "pig-nose" appearance of the duo- trasonography (IDUS). As with our previously reported case, we confirmed the utility of the "pignose" appearance and IDUS in the diagnosis of IPMN-associated pancreatobiliary fistulas. In addition, we found it difficult to manage biliary obstruction that resulted from the flow of mucinous material through pancreatobiliary fistulas. The obstruction was treated with endoscopic nasal biliary drainage (ENBD), but this was not always successful. In two of our cases, additional treatment with a large diameter fully covered metal stent failed to improve jaundice. Therefore, we conclude that standard endoscopic stenting may not be effective, and that alternative endoscopic methods or surgery may be necessary.

denal papilla in a case of pancreatobiliary fistula associated with IPMN [6]. In this paper, we report another three cases of IPMN-associated pancreatobiliary fistulas and discuss the role of "pignose" appearance and IDUS in the diagnosis of the disease.

\section{Case series}

$\nabla$

\section{Case 1}

In November 2010, an 84-year-old woman with cholangitis was admitted to our hospital, complaining of abdominal pain and anorexia. Contrast-enhanced (CE) CT showed multiple huge cystic lesions with septa, predominantly in the pancreatic head and body, and a suspected fistula connected to the CBD ( $\bullet$ Fig. 1a). Duodenal papilla images revealed dilated pancreatic and bile ducts with mucin extrusion (we call this "pignose" appearance) ( $\bullet$ Fig. 1b). Endoscopic retrograde cholangiopancreatography (ERCP) showed an extensive filling defect in the distal and mid CBD ( $\bullet$ Fig.1c). Subsequent IDUS showed a distinct pancreatobiliary fistula ( $\bullet$ Fig. 1 d), confirming the diagnosis of IPMN with a pancreatobiliary 

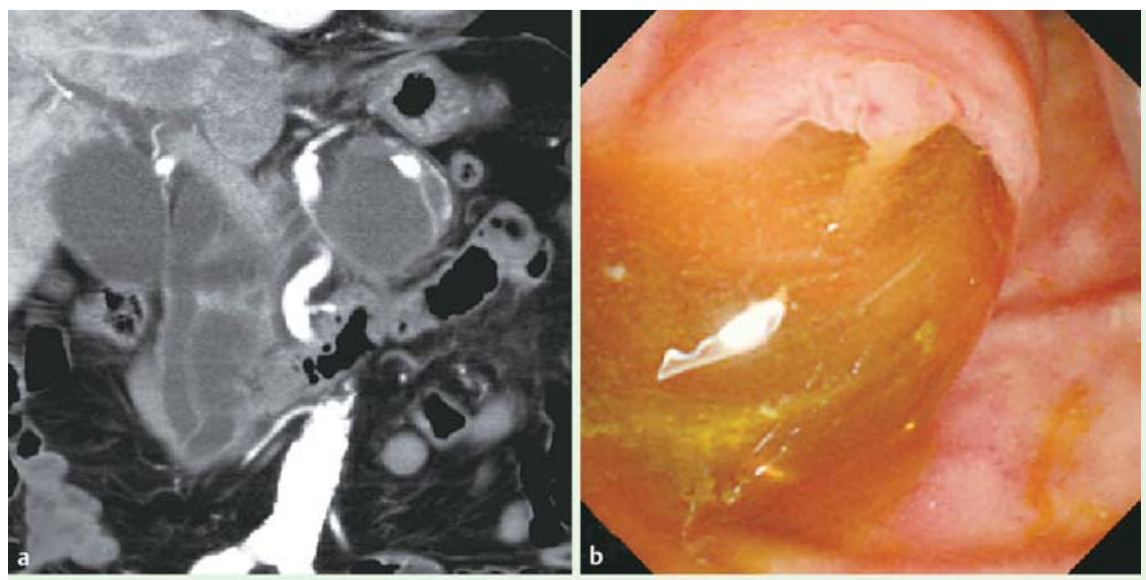

Fig. 1 a Computed tomography (CT) image showing multiple cystic lesions with septa in the pancreatic head and body, and a suspected fistula connecting to the common bile duct (CBD).

b Endoscopic appearance of the duodenal papilla: dilated pancreatic and bile ducts with mucin extrusion ("pig-nose" appearance). c Endoscopic cholangiogram revealing a dilated biliary tree in which the distal and mid CBD have an extensive filling defect. d Intraductal ultrasonography (IDUS) showing a pancreatobiliary fistula. The white arrows show the pancreatobiliary fistula. The white triangle shows the CBD.
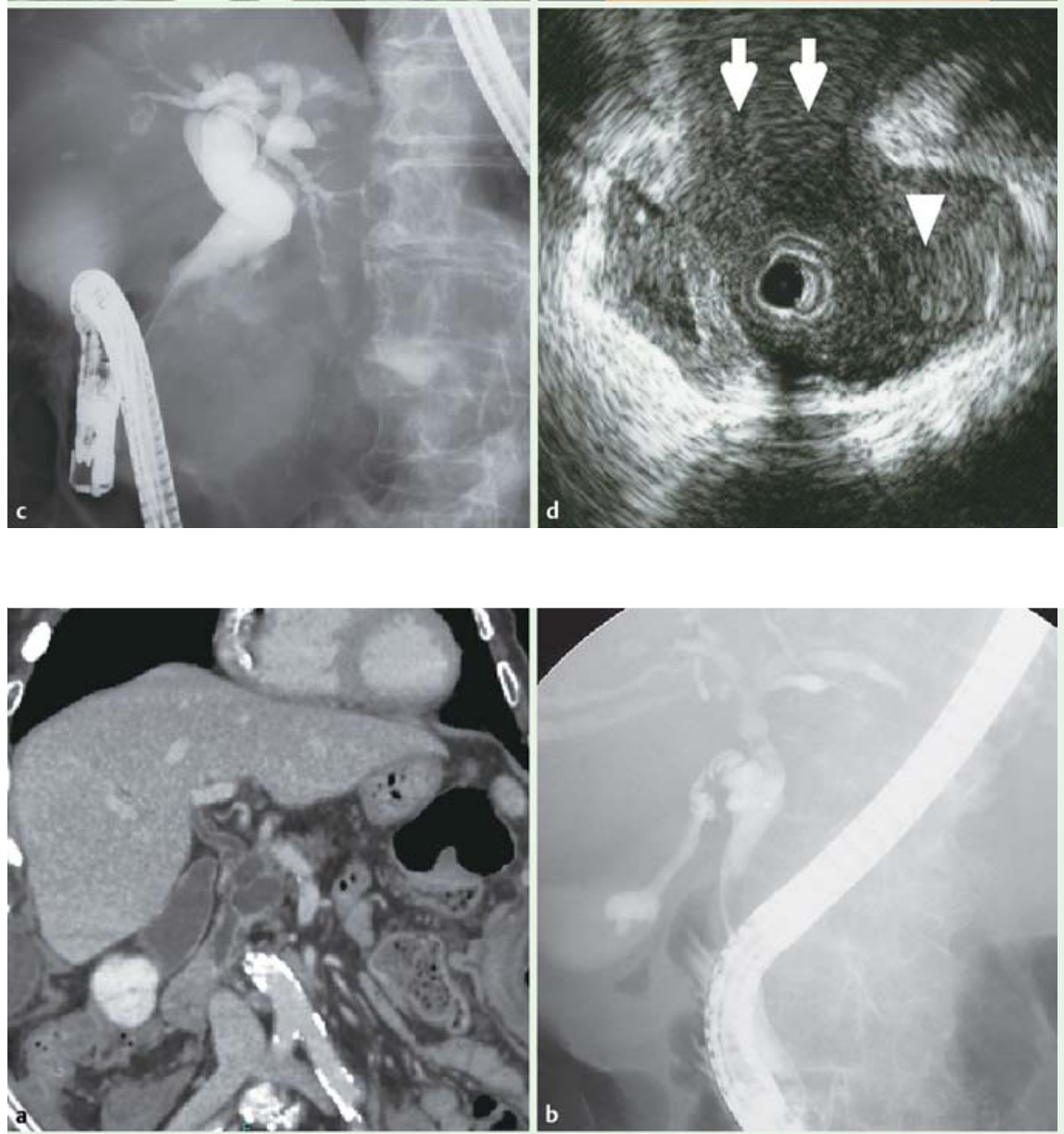

Fig. 2 a Computed tomography (CT) image showing a cystic lesion with multiple septa in the pancreatic head. b Endoscopic cholangiogram revealing a dilated biliary tree in which the mid common bile duct (CBD) has an extensive filling defect. c Intraductal ultrasonography (IDUS) showing a pancreatobiliary fistula. The white arrow shows the pancreatobiliary fistula. The white triangle shows the CBD. $\mathbf{d}$ Thick mucus plugs occluding the endoscopic nasal biliary drainage (ENBD) tube.
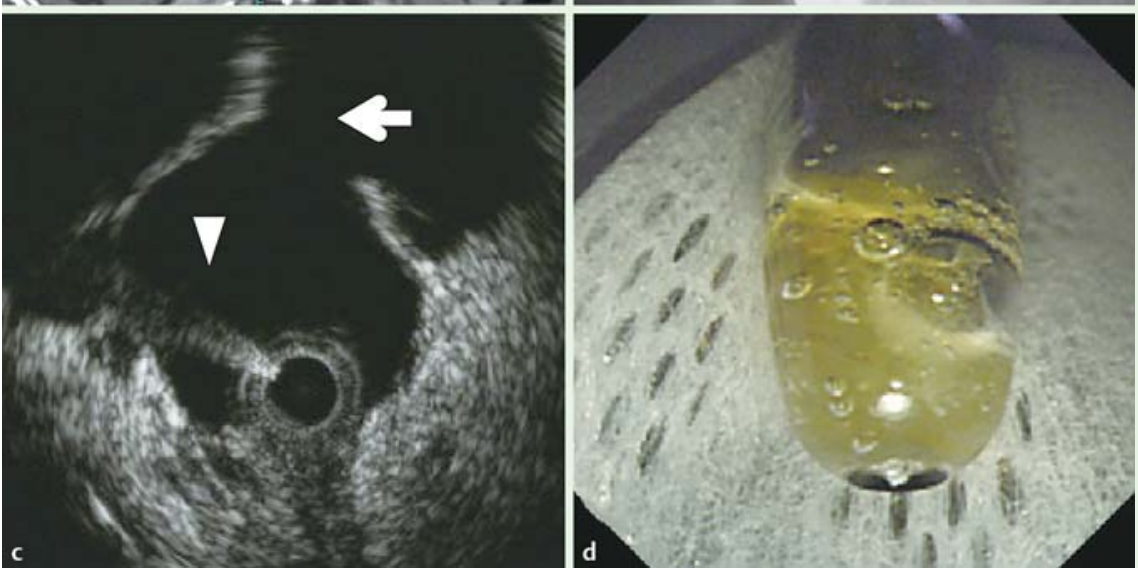

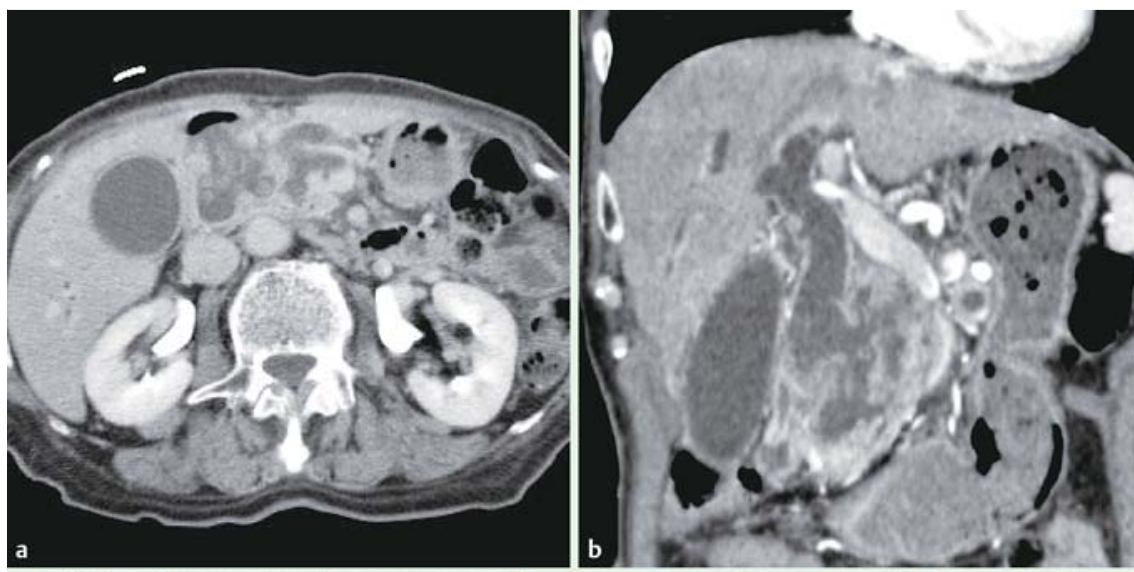

Fig. 3 a, b Computed tomography (CT) images showing a cystic lesion with multiple septa in the pancreatic head, and suspected fistulas connecting to the common bile duct (CBD) and duodenum. c Endoscopic appearance of the duodenum: ulcer with mucin extrusion. $\mathbf{d}$ Endoscopic appearance of the duodenal papilla: dilated pancreatic and bile ducts with mucin extrusion ("pig-nose" appearance). e Endoscopic cholangiogram revealing an extensive filling defect in the distal and mid CBD. f Intraductal ultrasonography (IDUS) showing a pancreatobiliary fistula. The white arrow shows the pancreatobiliary fistula. The white triangle shows the CBD.
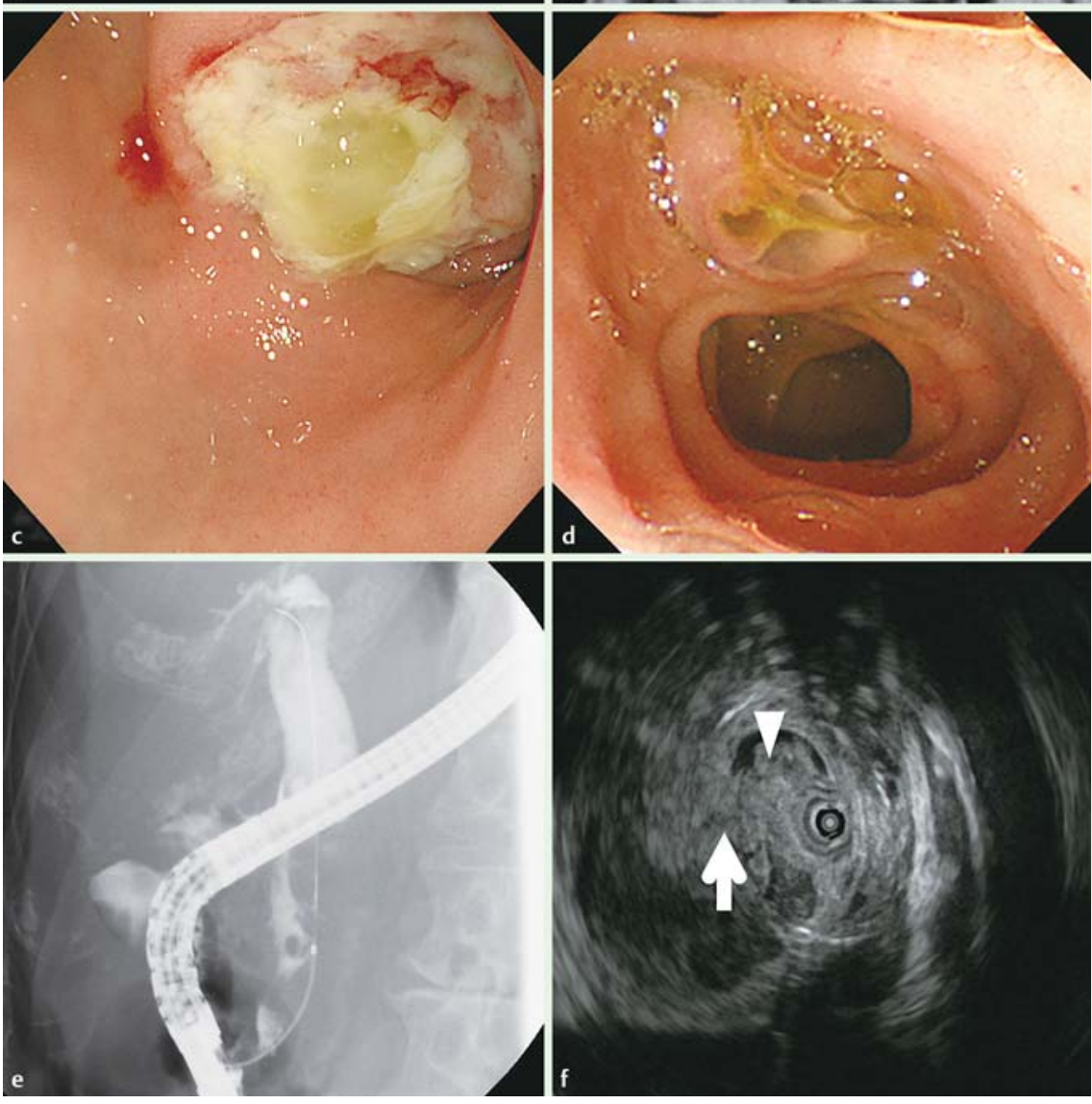

fistula. Insertion of a 7-French endoscopic nasal biliary drainage (ENBD) tube into the CBD followed by intermittent tube lavage led to improvement in cholangitis and jaundice. Then a fully covered biliary metallic stent was inserted for fistula coverage and biliary drainage. However, the patient's jaundice worsened. We considered a choledochojejunostomy, but she died 8 days later as a result of sudden worsening of cholangitis.

\section{Case 2}

In April 2011, an 87-year-old woman with cholangitis was admitted to our hospital, complaining of abdominal pain and fever. CECT showed a cystic lesion with multiple septa in the pancreatic head before the onset of cholangitis ( Fig. 2a). Plain CT did not detect the fistula and we suspected cholangitis due to CT negative stones. Subsequently, ERCP was performed and dilatation with an extensive filling defect was detected in the CBD ( $\bullet$ Fig.2b). Furthermore, IDUS revealed a perforation in the CBD that plain CT and
ERCP could not detect ( $\bullet$ Fig. 2 c). We diagnosed IPMN with a pancreatobiliary fistula and a 7-French ENBD tube was inserted into the CBD. However, it became occluded with thick mucus plugs 3 days later $(\bullet$ Fig. $2 \mathrm{~d}$ ). A thicker 8.5-French ENBD tube was inserted, but drainage was insufficient. The patient had another malignant disease with poor prognosis, so additional treatment was not provided before death.

\section{Case 3}

In June 2015, a 90-year-old man was admitted to our hospital complaining of abdominal pain. CECT showed a cystic lesion with multiple septa in the pancreatic head, and suspected fistulas connected to the CBD and duodenum ( $\bullet$ Fig. 3a,b). Esophagogastroduodenoscopy revealed a duodenal ulcer with mucin extrusion ( Fig. 3c). Duodenal papilla images revealed dilated pancreatic and bile ducts with mucin extrusion, the so-called "pig-nose" appearance ( $\bullet$ Fig.3d). ERCP showed an extensive filling defect 
Table 1 Summary of cases of intraductal papillary mucinous neoplasm (IPMN)-associated pancreatobiliary fistulas.

\begin{tabular}{|c|c|c|c|c|c|c|c|}
\hline \multirow[t]{2}{*}{ Case } & \multirow{2}{*}{$\begin{array}{l}\text { Age, years/ } \\
\text { gender }\end{array}$} & \multirow[t]{2}{*}{ Symptoms } & \multicolumn{3}{|c|}{ Identifiable fistula } & \multirow[t]{2}{*}{ "Pig-nose" appearance } & \multirow[t]{2}{*}{ ENBD effect } \\
\hline & & & CT & ERCP & IDUS & & \\
\hline Case 1 & $84 / \mathrm{F}$ & Abdominal pain/anorexia & Yes & Yes & Yes & Yes & Yes \\
\hline Case 2 & $87 / \mathrm{F}$ & Abdominal pain/fever & No & No & Yes & No & No \\
\hline Case 3 & $90 / \mathrm{M}$ & Abdominal pain & Yes & No & Yes & Yes & - \\
\hline Koizumi et al. [6] & $88 / \mathrm{F}$ & Abdominal and back pain & Yes & No & Yes & Yes & Yes \\
\hline
\end{tabular}

CT, computed tomography; ERCP, endoscopic retrograde cholangiopancreatography; IDUS, intraductal ultrasonography; ENBD, endoscopic nasal biliary drainage.

in the distal and mid CBD ( $\bullet$ Fig. 3e). Subsequent IDUS showed a distinct pancreatobiliary fistula ( $\bullet$ Fig. 3f), confirming the diagnosis of IPMN with a pancreatobiliary fistula. A fully covered biliary metallic stent was inserted, but migrated within a week. The fully covered biliary metallic stent was replaced above the sphincter of Oddi to prevent migration. However, the patient's jaundice did not improve. He chose to leave the hospital 10 days later.

\section{Discussion}

Fistula formation between the pancreas and adjacent organs has been reported in up to $6.6 \%$ of IPMN cases, with the duodenum, the $\mathrm{CBD}$, and the stomach being the most frequently affected sites $[2,3]$. IPMN-associated pancreatobiliary fistulas often develop obstructive jaundice and cholangitis, requiring urgent biliary drainage. However, diagnoses and details of IPMN-associated pancreatobiliary fistulas are not always given before surgery [4, 5]; therefore, improved diagnostic techniques are needed.

Including our previous report [6], we have observed four cases of IPMN-associated pancreatobiliary fistulas $($ Table 1$)$. In endoscopic evaluations, the duodenal papilla of IPMN patients is often described as having a "fish-mouth" appearance due to extrusion of mucinous material from the pancreatic duct into the small intestine [7]. However, in three of our four patients with IPMNassociated pancreatobiliary fistulas, mucinous material also extruded into the small intestine via the bile duct. The resulting "pig-nose" appearance of the duodenal papilla, in which both the bile duct and the pancreatic duct were dilated, may be the signature of pancreatobiliary fistulas associated with IPMN [6]. In the single case without "pig-nose" appearance, there was relatively little flow of mucinous extrusion into the bile duct. Therefore, although "pig-nose" appearance seems to be a unique marker for the diagnosis of IPMN-associated pancreatobiliary fistulas, it may not be present in all patients with this medical condition. Due to recent improvements in the resolution of CT and magnetic resonance imaging (MRI), these methods may detect IPMN-associated pancreatobiliary fistulas. However, we observed that plain CT may fail to detect pancreatobiliary fistulas (Case 2). Furthermore, contrast agents are preferably avoided in some cases because acute cholangitis often causes renal dysfunction. Our observations suggest that IDUS may be the best diagnostic option in certain circumstances. In particular, IDUS is optimal in two scenarios: (1) when ERCP fails to detect the fistula (as was observed in our three cases), because IDUS can be performed following ERCP, and (2) when "pig-nose" appearance is evident, because IDUS visualizes the CBD wall and its surrounding structures with higher resolution than CT and MRI. Therefore, IDUS is an ideal tool to evaluate pancreatobiliary fistulas.
In our three cases, biliary obstruction was initially treated with ENBD. Two of the three patients who were administered ENBD with intermittent ENBD tube lavage saw improvement in jaundice. The drainage effect was insufficient in one case (Case 2), in which the tip of the ENBD tube was located on the distal side of the pancreatobiliary fistula. It is important to note that ENBD with intermittent lavage is usually effective in improving jaundice. In our two cases, additional treatment with a large diameter fully covered metal stent failed to improve jaundice due to obstruction by mucinous material. Fully covered metallic stents (outer diameter: $10 \mathrm{~mm}$ ) were placed for the purpose of closing the fistula; however, because the CBD diameter was larger than $10 \mathrm{~mm}$, the stents failed to stop the flow of mucus from the pancreatobiliary fistula into the CBD. The placement of a large diameter stent to prevent blockage by mucus seems insufficient compared to ENBD or percutaneous transhepatic biliary drainage (PTBD), which may actually remove the mucus after intermittent lavage. However, ENBD and PTBD tubes may not be suitable for long-term placement. Moreover, in cases like these, there may be a better option for treating IPMN-associated pancreatobiliary fistulas: surgery [2]. A case study reported by Patel et al. noted that a patient who eventually underwent surgical biliary bypass achieved successful long-term relief of cholestasis [8]. Therefore, surgical options should be considered in patients who are tolerant to resection and who become jaundiced despite biliary drainage $[2,8]$. Moreover alternative endoscopic methods (such as placement of a fully covered metallic stent larger than the CBD diameter, or multiple metal biliary stents, or endoscopic ultrasound (EUS)-guided transduodenal choledochoduodenostomy proximal to the fistula with a fully covered metal stent, or EUSguided hepaticogastrostomy with a fully covered metal stent) may be effective.

We have drawn three main conclusions from our case studies. First, we have confirmed the "pig-nose" appearance of the duodenal papilla as a unique marker that can be used to diagnose IPMN-associated pancreatobiliary fistulas. Second, IDUS enables us to confirm diagnoses of IPMN-associated pancreatobiliary fistulas, and ENBD following IDUS should be considered in patients before additional treatment. Third, when ENBD is insufficient, alternative endoscopic methods or surgery may be necessary.

\section{Competing interests: None}




\section{References}

1 Ohashi K, Murakami Y, Maruyama M et al. Four cases of mucin producing cancer of the pancreas on specific findings of the papilla of Vater. [in Japanese]. Prog Dig Endosc 1982; 20: 348 - 351

2 Kurihara K, Nagai H, Kasahara Ket al. Biliopancreatic fistula associated with intraductal papillary mucinous pancreatic cancer: institutional experience and review of the literature. Hepatogastroenterology 2000; 47: 1164-1167

3 Kobayashi G, Fujita N, Noda Y et al. Intraductal papillary mucinous neoplasms of the pancreas showing fistula formation into other organs. J Gastroenterol 2010; 45: 1080-1089

4 Okada K, Furuuchi T, Tamada T et al. Pancreatobiliary fistula associated with an intraductal papillary-mucinous pancreatic neoplasm mani- festing as obstructive jaundice: report of a case. Surg Today 2008; 38 : $371-376$

5 Sano S, Nishimori I, Okamoto $N$ et al. Biliopancreatic fistula caused by an intraductal papillary-mucinous tumor of the pancreas confirmed by biochemical analysis of mucin. Int J Gastrointest Cancer 2003; 34: $101-106$

6 Koizumi M, Kumagi T, Hiasa Y. An unusual cause of abdominal pain. Gastroenterology 2015; 148: e1 -e2

7 Retter J, Dinter D, Bersch C et al. Acute recurrent pancreatitis curtaining an intraductal papillary mucinous tumor of the pancreas. J Gastrointestin Liver Dis 2007; 16: 445-447

8 Patel A, Lambiase L, Decarli A et al. Management of the mucin filled bile duct. A complication of intraductal papillary mucinous tumor of the pancreas. JOP 2005; 6: 255-259 